\title{
HIV-1 Tat-induced diarrhea is improved by the PPARalpha agonist, palmitoylethanolamide, by suppressing the activation of enteric glia
}

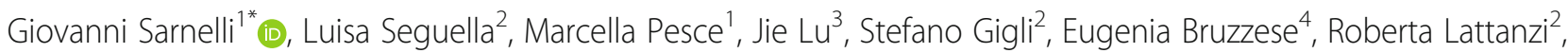
Alessandra D'Alessandro', Rosario Cuomo ${ }^{1}$, Luca Steardo ${ }^{1,2}$ and Giuseppe Esposito ${ }^{2^{*}}$

\begin{abstract}
Background: Diarrhea is a severe complication in HIV-1-infected patients with Trans-activator of transcription (HIV-1 Tat) protein being recognized as a major underlying cause. Beside its direct enterotoxic effects, Tat protein has been recently shown to affect enteric glial cell (EGC) activity. EGCs regulate intestinal inflammatory responses by secreting pro-inflammatory molecules; nonetheless, they might also release immune-regulatory factors, as palmytoilethanolamide (PEA), which exerts anti-inflammatory effects by activating PPARa receptors. We aimed at clarifying whether EGCs are involved in HIV-1 Tat-induced diarrhea and if PEA exerts antidiarrheal activity.
\end{abstract}

Methods: Diarrhea was induced by intracolonic administration of HIV-1 Tat protein in rats at day 1. PEA alone or in the presence of peroxisome proliferator-activated receptor (PPAR) antagonists was given intraperitoneally from day 2 to day 7. S100B, iNOS, NF-kappaB, TLR4 and GFAP expression were evaluated in submucosal plexi, while S100B and NO levels were measured in EGC submucosal plexi lysates, respectively. To verify whether PEA effects were PPARa-mediated, PPARa ${ }^{-1-}$ mice were also used. After 7 days from diarrhea induction, endogenous PEA levels were measured in submucosal plexi homogenates deriving from rats and $\mathrm{PPARa}^{-/-}$mice.

Results: HIV-1 Tat protein induced rapid onset diarrhea alongside with a significant activation of EGCs. Tat administration significantly increased all hallmarks of neuroinflammation by triggering TLR4 and NF-kappaB activation and S100B and iNOS expression. Endogenous PEA levels were increased following HIV-1 Tat exposure in both wildtype and knockout animals. In PPARa ${ }^{-1-}$ mice, PEA displayed no effects. In wildtype rats, PEA, via PPARadependent mechanism, resulted in a significant antidiarrheal activity in parallel with marked reduction of EGCsustained neuroinflammation.

Conclusions: EGCs mediate HIV-1 Tat-induced diarrhea by sustaining the intestinal neuroinflammatory response. These effects are regulated by PEA through a selective PPARa-dependent mechanism. PEA might be considered as an adjuvant therapy in HIV-1-induced diarrhea.

Keywords: HIV-1 Tat protein, EGCS, Diarrhea, Neuroinflammation, PEA

\footnotetext{
*Correspondence: sarnelli@unina.it; giuseppe.esposito@uniroma1.it

'Department of Clinical Medicine and Surgery, "Federico II" University of

Naples, 80131 Naples, Italy

2Department of Physiology and Pharmacology, "Vittorio Erspamer", La

Sapienza University of Rome, 00185 Rome, Italy

Full list of author information is available at the end of the article
} 


\section{Background}

The use of combined anti-retroviral therapy against human immunodeficiency virus-type 1 (HIV-1) infection has dramatically improved the survival and prognosis of patients affected by the acquired immunodeficiency syndrome (AIDS) [1]. However, chronic diarrhea is reported in up to $30 \%$ of HIV-1-infected patients and significantly contributes to AIDS morbidity [2, 3].

Many of the pathogenic effects of HIV-1 in the gut are caused by the HIV-1 trans-activating factor protein (Tat), a viral protein of 86 aminoacids, which is essential to viral replication [4]. HIV-1 Tat targets enterocytes and induces the expression of many genes regulating cells' survival and growth; but it also affects immune and inflammatory responses, altering the intracellular calcium concentration, inducing epithelial cell apoptosis, and ultimately causing secretory diarrhea $[5,6]$. The intestinal epithelial mucosa has been considered for years as the key target in HIV-1 Tat-related enterotoxicity; however, the enteric nervous system (ENS) is now emerging to be also involved [7, 8]. Within the ENS, enteric glial cells (EGCs), together with neurons, cooperate to finely regulate secretion, motility, and blood flow, as well as immune responses [9-11]. EGCs express Toll-like receptors (TLRs), and there is mounting evidence suggesting that they actively participate to the homeostatic immune control of the gut [12]. EGCs are able to secrete pro-inflammatory mediators, interleukins, and enteroglial-released factors [13-15], but they also release a number of protective mediators that sustain epithelial barrier functions [16-18]. Among EGC-derived factors, S100B, a specific glial $\mathrm{Ca}^{+2} / \mathrm{Zn}^{+2}$-binding protein, and nitric oxide (NO) deriving from the inducible isoform of nitric oxide synthase (iNOS) expressed by EGCs play a central role during immune-inflammatory responses $[14,19,20]$. We recently demonstrated that glial cells participate to HIV-1 Tat-induced intestinal and neurological pathogenesis [21], but the possibility to pharmacologically modulate Tatinduced secretory diarrhea by inhibiting EGC activation has not been explored yet.

Palmitoylethanolamide (PEA), an endogenous, on-demand released N-Acylethanolamide [22-24], exerts immunoregulatory functions targeting EGC activation in ulcerative colitis, with a consistent protection of colonic epithelial mucosa [25]. Different studies have shown that the pharmacological activity of PEA depends on its capacity to selectively bind peroxisome proliferatoractivated receptor- $\alpha$ (PPAR $\alpha)$, a member of a nuclear hormone receptor superfamily of ligand-activated transcription factor [24]. The involvement of and the ability of PEA to protect against HIV-1 Tat-induced diarrhea have never been investigated.

The aims of the present study were to investigate the involvement of EGCs in a rat model of diarrhea induced by the intracolonic administration of $\mathrm{HIV}-1$ Tat, to characterize the mediators secreted by EGCs activation, and to evaluate the protective effect of PEA, its site, and mechanisms of action, respectively.

\section{Methods \\ Animals and experimental design}

Eight-week-old Wistar male rats (Harlan Laboratories, Udine, Italy) and 6-week-old PPAR $\alpha^{-1-}$ mice (Taconic, Germantown, New York, USA) were used for experiments. All procedures were approved by La Sapienza University's Ethics Committee. Animal care was in compliance with the IASP and European Community (EC L358/1 18/12/86) guidelines on the use and protection of animals in experimental research. Rats were randomly divided into the following groups ( $n=8$ each): non-diarrhea, vehicle group; HIV-1 Tat protein-induced diarrhea group; HIV-1 Tat protein-induced diarrhea group receiving daily PEA 2 and $10 \mathrm{mg} / \mathrm{Kg}$, respectively; HIV-1 Tat protein-induced diarrhea group receiving daily PEA $(10 \mathrm{mg} / \mathrm{Kg})$ and selective PPAR $\alpha$ antagonist MK866 (10 mg/Kg) and selective PPAR $\gamma$ antagonist GW9662 (1 mg/Kg), respectively; HIV-1 Tat proteininduced diarrhea group receiving $0.03 \% \mathrm{w} / \mathrm{v}$ lidocaine; bisacodyl group $(20 \mathrm{mg} / \mathrm{Kg})$ as internal control in some experiments. Analogously, PPAR $\alpha^{-/-}$mice were randomly divided into the following groups $(n=8$ each): non-diarrhea, vehicle group; HIV-1 Tat protein-induced diarrhea group; HIV-1 Tat protein-induced diarrhea group receiving daily PEA 50 and $100 \mathrm{mg} / \mathrm{Kg}$, respectively; HIV-1 Tat protein-induced diarrhea group receiving $0.03 \% w / v$ lidocaine; bisacodyl group $(20 \mathrm{mg} / \mathrm{Kg})$ as internal control in some experiments.

Diarrhea was experimentally induced by intracolonic administration of HIV-1 Tat protein $(130 \mathrm{ng} / \mathrm{Kg})$ at day 1. HIV-1 Tat was dissolved in pyrogen-free distillated water and a volume of $400 \mu \mathrm{l}$ or $40 \mu \mathrm{l}$ of a $100 \mathrm{ng} / \mathrm{ml} \mathrm{so-}$ lution of HIV-1 Tat was injected into the lumen of the rat and PPAR $\alpha^{-1-}$ mice colon (3-4 $\mathrm{cm}$ proximal to anus) by using a 24-gauge catheter, respectively. PEA alone, or combined with PPARs antagonists, was given intraperitoneally from day 1 to day 7 .

In a subset of experiments, lidocaine hydrochloride monohydrate (Sigma-Aldrich, Milan, Italy, 0.03\% w/v) dissolved in sterile, pyrogen-free distilled water, was given in a single dose through intracolonic administration at day 1 concomitantly with HIV-1 Tat. In another set of experiments, bisacodyl was administrated orally as aqueous solution at day 1 and served as positive internal control. Depending upon the experimental plan, at day 7 , animals were euthanatized and colon was isolated to perform macroscopic, histochemical, and biochemical analyses as described below.

\section{Evaluation of diarrhea}

Depending upon the experimental protocol, animals were separated in subgroups and placed separately in cages lined 
with filter paper to evaluate diarrhea severity. The individual cages were inspected every $2 \mathrm{~h}$ for $16 \mathrm{~h}$, from day 1 to day 7 after HIV 1-Tat intracolonic administration, for the presence of characteristic wet diarrhoeal droppings. Daily defecation frequency and number of unformed water fecal pellets of each animal were assessed and compared with the score from the vehicle group. The data were expressed as a daily mean score $(16 \mathrm{~h})$ of diarrhoeal dropping number and total number of fecal pellets/wet spots for defecation frequency within 7 days from diarrhea induction. Evaluation of accumulation of intracolonic fluid was performed using the enteropooling technique according to previously described method [26]. Briefly, enteropooling is defined as the intraluminal accumulation of fluid into the small intestine and corresponds with the fluid already located in the lumen and excreted from the blood. According to the experimental plan, the entire small intestine and colon of rats and PPAR ${ }^{-1-}$ mice were isolated, taken care to avoid tissue rupture and loss of fluid, by removing the mesentery and connective tissue. To normalize the data, fluid accumulation was expressed as follows:

$$
\left(W_{1}-W_{2}\right) / W_{2} \times 10^{-6}
$$

where $W_{1}$ is the weight of the intestine after excision and $W_{2}$ is the weight of the intestine after expulsion of its content. Water content was measured and compared with the score from vehicle group.

\section{Tissue preparation}

To isolate submucosal plexi from animals at day 7 after diarrhea induction, we performed a slightly modified method than previously described procedure by Cirillo et al. [14]. Following dissection, colonic segments (approximately 2-cm long) were collected and placed in a cold oxygenated sterile Krebs solution containing (in mM) 117 $\mathrm{NaCl}, 4.7 \mathrm{KCl}, 1.2 \mathrm{MgCl}_{2} 6 \mathrm{H}_{2} \mathrm{O}, 1.2 \mathrm{NaH}_{2} \mathrm{PO}_{4}, 25$ $\mathrm{NaHCO}_{3}, 2.5 \mathrm{CaCl}_{2} 2 \mathrm{H}_{2} \mathrm{O}$, and 11 glucose under carbogen $\left(5 \% \mathrm{CO}_{2}, 95 \% \mathrm{O}_{2}\right)$ atmosphere equilibrated at $\mathrm{pH}$ 7.4. The tissue was longitudinally cut along the mesenteric border, and the submucosal plexus was carefully separated from the mucosal and the muscle layers by microdissection. After removal, submucosal plexi were processed for biochemical and immunofluorescence assays.

\section{Protein extraction and western blot analysis}

Proteins were extracted from submucosal plexi deriving from rats and PPAR $\alpha^{-1-}$ mice at day 7 after diarrhea induction. The tissue was homogenized in ice-cold hypotonic lysis buffer to obtain cytosolic extracts and underwent electrophoresis through a polyacrilamide minigel. Proteins were transferred into nitrocellulose membrane that were saturated with non-fat dry milk and then incubated with either mouse anti-S100B (Neo-
Marker, Milan, Italy), mouse anti-iNOS, rabbit antiGFAP, rabbit anti-TLR4, and mouse anti- $\beta$-actin (all Santa Cruz Biotechnology, Santa Cruz, California, USA). Membranes were then incubated with the specific secondary antibodies conjugated to horseradish peroxidase (Dako, Milan, Italy). Immune complexes were revealed by enhanced chemiluminescence detection reagents (Amersham Biosciences, Milan, Italy). Blots were analyzed by scanning densitometry (GS-700 imaging densitometer; Bio-Rad). Results were expressed as OD (arbitrary units; $\mathrm{mm}^{2}$ ) and normalized on the expression of the housekeeping protein $\beta$-actin.

\section{Electrophoretic mobility shift assay (EMSA)}

EMSA was performed to detect NF-kappaB activation in submucosal plexi obtained from rats and PPAR ${ }^{-1-}$ mice at day 7 after diarrhea induction. Double-stranded oligonucleotides containing the NF-kappaB recognition sequence for rats (5-CAACGG CAGGGGAATCTCCCTCTCCTT-3) and mice (5-TCAGAGGGGACTTTCCGAGAGG-3) were end-labeled with ${ }^{32} \mathrm{P} \gamma$-ATP. Nuclear extracts were incubated for $15 \mathrm{~min}$ with radiolabeled oligonucleotides (2.5$\left.5.0 \times 10^{4} \mathrm{cpm}\right)$ in $20 \mathrm{ml}$ reaction buffer containing $2 \mathrm{mg}$ poly dI-dC, $10 \mathrm{mM}$ Tris- $\mathrm{HCl}$ (pH 7.5), $100 \mathrm{mM} \mathrm{NaCl}$, $1 \mathrm{mM}$ ethylenediaminetetraacetic acid, $1 \mathrm{mM}$ dl-dithiothreitol, $1 \mathrm{mg} / \mathrm{ml}$ bovine serum albumin, and $10 \%(v / v)$ glycerol. Nuclear protein-oligonucleotide complexes were resolved by electrophoresis on a $6 \%$ non-denaturing polyacrylamide gel in 1 Tris Borate ethylenediaminetetraacetic acid buffer at $150 \mathrm{~V}$ for $2 \mathrm{~h}$ at $4{ }^{\circ} \mathrm{C}$. The gel was dried and autoradiographed with an intensifying screen at $-80{ }^{\circ} \mathrm{C}$ for $20 \mathrm{~h}$. Subsequently, the relative bands were quantified by densitometric scanning with Versadoc (Bio-Rad Laboratories) and a computer program (Quantity One Software, Bio-Rad Laboratories). ${ }^{32} \mathrm{P}-\gamma$-ATP was from Amersham (Milan, Italy). Poly dI-dC was from Boehringer-Mannheim (Milan, Italy). Oligonucleotide synthesis was performed to our specifications by Tib Molbiol (Boehringer-Mannheim).

\section{NO quantification}

$\mathrm{NO}$ was measured as nitrite $\left(\mathrm{NO}_{2}^{-}\right)$accumulation in submucosal plexi homogenates deriving from rats and PPAR $^{-1-}$ mice at day 7 after diarrhea induction, by a spectrophotometer assay based on the Griess reaction. Briefly, Griess reagent (1\% sulphanilamide, 0.1\% naphthylethylenediamine in phosphoric acid) was added to an equal volume of supernatant, and the absorbance was measured at $550 \mathrm{~nm}$. Nitrite concentration (nM) was thus determined using a standard curve of sodium nitrite.

\section{Enzyme-linked immunosorbent assay for S100B}

Enzyme-linked immunosorbent assay (ELISA) for S100B (Biovendor R\&D, Brno, Czech Republic) was carried out on submucosal plexi lysates obtained from rats and 
PPARK $^{-1-}$ mice at day 7 after diarrhea induction, according to the manufacturer's protocol. Absorbance was measured on a microtiter plate reader. S100B level was determined using standard curves method.

\section{Immunofluorescence analysis}

Additional experiments were performed using specific isotype antibody controls (Abcam, Cambridge, UK), at the same concentration as the primary antibodies. Tissues were then incubated in the dark with the proper secondary antibody: fluorescein isothiocyanate-conjugated anti-rabbit or Texas Red-conjugated anti-mouse, respectively (both Jackson ImmunoResearch Laboratories, West Grove, PA, USA). Tissues were analyzed with a microscope (Nikon Eclipse 80i), and images were captured by a high-resolution digital camera (Nikon Digital Sight DS-U1).

\section{Measurement of PEA in rats and mice EGCs}

Tissue content of endogenous PEA was measured in submucosal plexi homogenates deriving from both rats and PPAR ${ }^{-1-}$ mice at day 7 after diarrhea induction. Following isolation of lipidic fraction by tissue homogenates, intracellular PEA concentrations (pmol) were normalized per milligram of extracted lipid fraction and were analyzed by liquid chromatography coupled to tandem mass spectrometry (LC-MS/MS) using a 325-MS LC/MS Triple Quadrupole Mass Spectrometer (Agilent Technologies Italia, Cernusco $\mathrm{s} / \mathrm{N}$, Italy) according to literature [27].

\section{Statistical analysis}

Results were expressed as mean \pm SEM of $n$ experiments. A statistical analysis was performed using parametric one-way analysis of variance (ANOVA), and multiple comparisons were performed by Bonferroni's post hoc test; $p$ values $<0.05$ were considered significant.

\section{Results}

Intracolonic administration of HIV-1 Tat induces diarrhea in rats and stimulates the release of endogenous PEA Intracolonic administration of HIV-1 Tat-induced acute diarrhea, starting from day 1 and lasting up to 7 days post treatment; the severity of which was significantly improved by PEA, in a concentration-dependent fashion and in PPAR $\alpha$-dependent manner (Fig. 1). Interestingly, the antidiarrheal effect of PEA appeared to be specifically related to HIV-1 Tat administration since PEA treatment failed to significantly inhibit bisacodyl-induced diarrhea, even at the highest dose (Additional file 1).

In order to provide a rational for the antidiarrheal effect of PEA, we measured its intracellular content in submucosal plexi after 7 days from the induction of the diarrhea. We found that HIV-1 Tat significantly increased the levels of endogenous PEA and that this increase was significantly inhibited by lidocaine $(+147$ and $-50 \%$ vs. vehicle group and HIV-1 Tat group, respectively; all $p<0.01$; Fig. 2); in rats receiving bisacodyl, the tissue content of PEA was unaffected, likely suggesting that it is dependent by the activation of EGCs.

\section{HIV-1 Tat induces an inflammatory response through EGC} activation that is inhibited by PEA

Administration of HIV-1 Tat induced a significant activation of NF- $\mathrm{KB}$ and resulted in an increased expression of GFAP, S100B, TLR4, and iNOS in rat submucosal plexi (Fig. 3, all $p<0.001$ vs. vehicle group); significantly, the $\mathrm{S} 100 \mathrm{~B}$ and nitrite levels were also increased.

PEA treatment significantly reduced HIV-1 Tat-induced NF- $\kappa B$ activation, in a dose-dependent manner $(-42$ and $-58 \%, p<0.01$ and $p<0.001)$; similarly, the activation of EGCs was inhibited by PEA, with the expression of GFAP ( -35 and $-69 \%)$, S100B ( -29 and $-65 \%)$, TLR4 (- 31 and $-70 \%$ ), iNOS (- 35 and $74 \%$ ), and the levels of S100B $(-27$ and $-64 \%)$ and nitrite $(-30$ and $-64 \%)$ being all significantly reduced $(p<0.01$ and $p<0.001$; at 2 and $10 \mathrm{mg} / \mathrm{kg}$, respectively; Fig. 3). The ability of PEA to reduce HIV-1 Tat-induced EGC activation was significantly inhibited by MK866, but not by GW9662, suggesting that its effect was mediated by PPAR- $\alpha$ rather than by PPAR- $\gamma$. PEA failed to significantly affect the release of EGCderived mediators in rats with bisacodyl-induced diarrhea (Fig. 3), and this finding suggests that its effect specifically targets HIV-1 Tat-induced diarrhea by acting on EGCrelated activation.

Further supporting the involvement of EGCs, a significantly higher S100B/iNOS expression was observed in the submucosal plexi preparation of HIV-1 Tat-treated rats, compared to vehicle group (+310 and $+260 \%$, respectively, $p<0.001$ ) (Fig. 4). Treatment with PEA (2$10 \mathrm{mg} / \mathrm{kg}$ ) reduced the expression of both markers, in a concentration-dependent manner $(-28$ and $-50 \%,-26$ and $-40 \%$ for S100B and iNOS, respectively; $p<0.01$ and $p<0.001$ vs. HIV-1 Tat; Fig. 4). Similar to what was reported above, the effect of PEA was inhibited by the presence of the PPAR $\alpha$-, but not PPAR $\gamma$-antagonist, while bisacodyl administration failed to modify S100B/ iNOS expression (Fig. 4).

PEA treatment failed to improve HIV-1 Tat-induced diarrhea and EGC-associated neuroinflammation in PPARa ${ }^{-/-}$mice Intracolonic administration of HIV-1 Tat protein induced acute diarrhea in PPARo ${ }^{-1-}$ mice, similar to what was observed in a subset of wild mice (data not shown) and in rats (Additional file 2); the diarrhea was associated with the increased activation of NF-kappaB of submucosal enteric glial cells and the upregulation of S100B/iNOS expression (Fig. 5). In line with the results obtained with the PPAR $\alpha$ antagonist, treatment with 


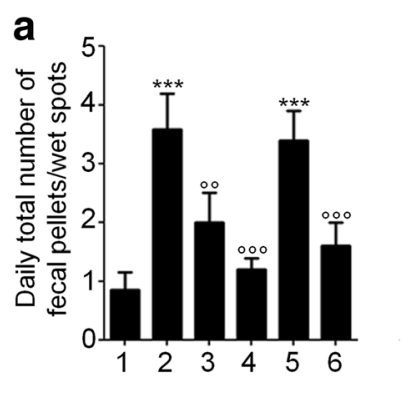

b

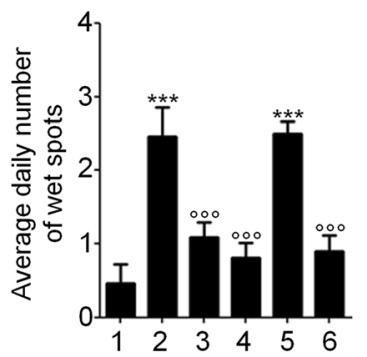

1. Vehicle

2. HIV-1 Tat $130 \mathrm{ng} / \mathrm{Kg}$

3. HIV-1 Tat $130 \mathrm{ng} / \mathrm{Kg}+$ PEA $2 \mathrm{mg} / \mathrm{Kg}$

4. HIV-1 Tat $130 \mathrm{ng} / \mathrm{Kg}+$ PEA $10 \mathrm{mg} / \mathrm{Kg}$

5. HIV-1 Tat $130 \mathrm{ng} / \mathrm{Kg}+\mathrm{PEA} 10 \mathrm{mg} / \mathrm{Kg}+\mathrm{MK} 86610 \mathrm{mg} / \mathrm{Kg}$

6. HIV-1 Tat $130 \mathrm{ng} / \mathrm{Kg}+$ PEA $10 \mathrm{mg} / \mathrm{Kg}+\mathrm{GW} 96621 \mathrm{mg} / \mathrm{Kg}$
C

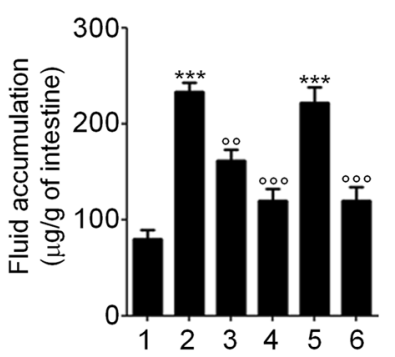

Fig. 1 Palmitoylethanolamide (PEA) improves diarrheal hallmarks in rats via PPARa activation. Intracolonic administration of HIV-1 Tat protein $(130 \mathrm{ng} / \mathrm{Kg})$ resulted in a significant increase of $\mathbf{a}$ daily defecation frequency, $\mathbf{b}$ average daily number of wet spots, and $\mathbf{c}$ fluid accumulation within 7 days from diarrhea induction. Administration of PEA significantly improved diarrhea in a concentration-dependent manner (at 2 and $10 \mathrm{mg} / \mathrm{kg}$, respectively); the antidiarrheal activity of PEA was significantly inhibited in the presence of PPARa antagonist (MK866), whereas PPARY antagonist (GW9662) had no effect. The results are expressed as mean \pm SEM of $n=5$ experiments. ${ }^{* *} p<0.001$ vs. vehicle group; ${ }^{\circ 0} p<0.01$ and ${ }^{\circ 0}$ ${ }^{\circ} p<0.001$ vs. HIV-1 Tat group

PEA, even at highest doses, failed to significantly improve the diarrhea in PPAR ${ }^{-1-}$ mice and had no effect on the activation of EGCs induced by HIV-1 Tat (Fig. 5).

Bisacodyl treatment was able to induce the diarrhea in PPARo ${ }^{-/-}$mice, but was unaffected by the administration of PEA (Fig. 5). Bisacodyl failed to modify the tissue content of endogenous PEA which was, conversely, significantly increased by HIV-1 Tat administration and inhibited by lidocaine $(+250 \%$ vs. vehicle group, and $-55 \%$ vs. HIV-1 Tat group, respectively; all $p<0.01$; Fig. 5).

\section{Discussion}

Secretory diarrhea is a common clinical issue observed in nearly $60-80 \%$ of HIV-1 patients, and it is considerably widespread in third-world countries [28]. So far, HIV-1 Tat protein has been identified as the main responsible for the damage of intestinal mucosal, by promoting pro-oxidant and proapoptotic-mediated disruption of colonic epithelial cells and consequently the intestinal barrier integrity [5, 29]. More recently, it has been described that HIV-1 Tat protein has an additional effect on the

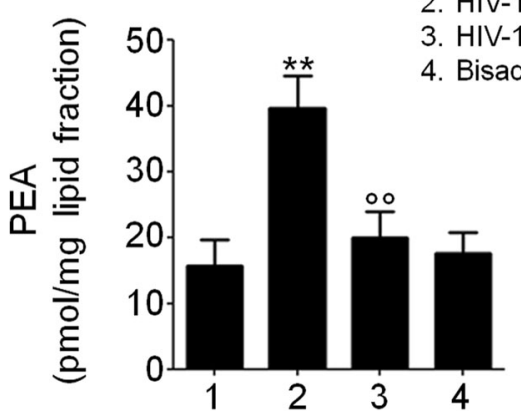

Fig. 2 HIV-1 Tat administration increases endogenous PEA levels in submucosal plexi EGCs. As a consequence of HIV-1 Tat intracolonic administration, a marked increase of PEA produced in rat submucosal plexi was observed in comparison with vehicle group. Lidocaine $(0.03 \% \mathrm{~W} / \mathrm{V})$ counteracted this HIV-1 Tat-induced effect, while bisacodyl treatment did not induce any significant changes in PEA levels. The results are expressed as mean \pm SEM of $n=5$ experiments. ${ }^{* *} p<0.01$ vs. vehicle group; ${ }^{\circ 0} p<0.01$ vs. HIV-1 Tat group 


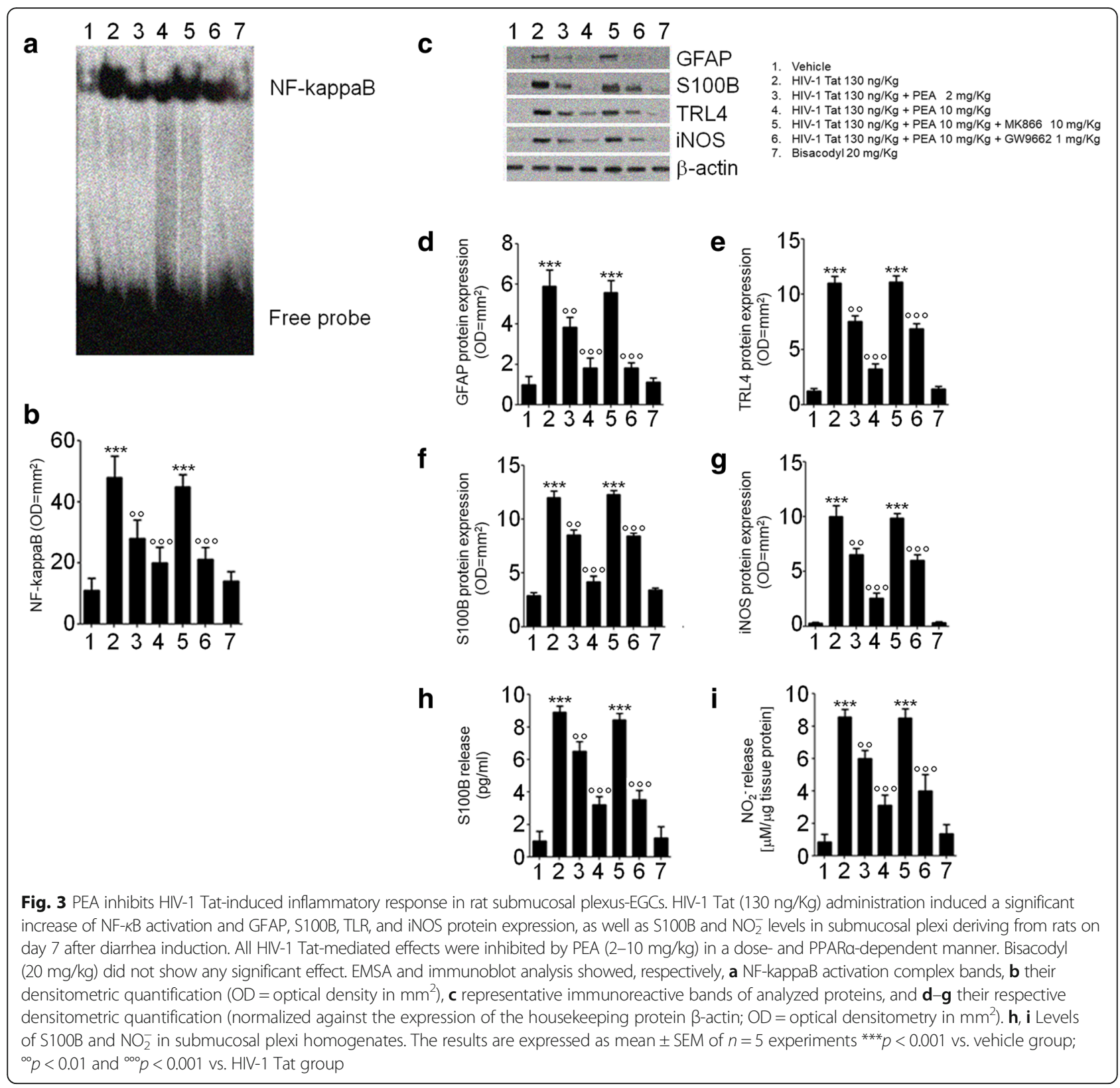

nervous part of the gut, the ENS [7, 21, 30]. This direct action on the nervous system, which regulates many intestinal functions, causes abnormalities in neuronal excitability that, together with the release of pro-inflammatory cytokines in the intestinal milieu, contributes to gut dysfunction described in HIV patients [7].

Our results demonstrate that, beside its effect on enteric neurons, HIV-1 Tat protein also targets EGCs and mediates the overexpression of specific glial markers, as S100B and GFAP, in colonic submucosal plexus with a parallel increase in the expression of iNOS protein and pro-inflammatory signaling molecules (i.e., $\mathrm{NO}$ ).
These final events occur via activation of the NFkappaB-mediated cascade and TLR4 activation, two molecular pathways that are linked to each other during EGC activation [25].

The ability of the ENS to modulate virus-induced diarrhea was first reported by Lundgren et al., who showed that the inhibition of enteric nerves excitability was able to significantly inhibit the diarrhea induced by rotavirus [31]. Accordingly, we showed that lidocaine challenge was able to dampen symptoms and biochemical markers indicative for secretory diarrhea, further supporting the role of the ENS, as a whole, in mediating HIV-1 Tatinduced diarrhea. We here show that enteric glia cells take part in mediating the diarrhea induced by viral 

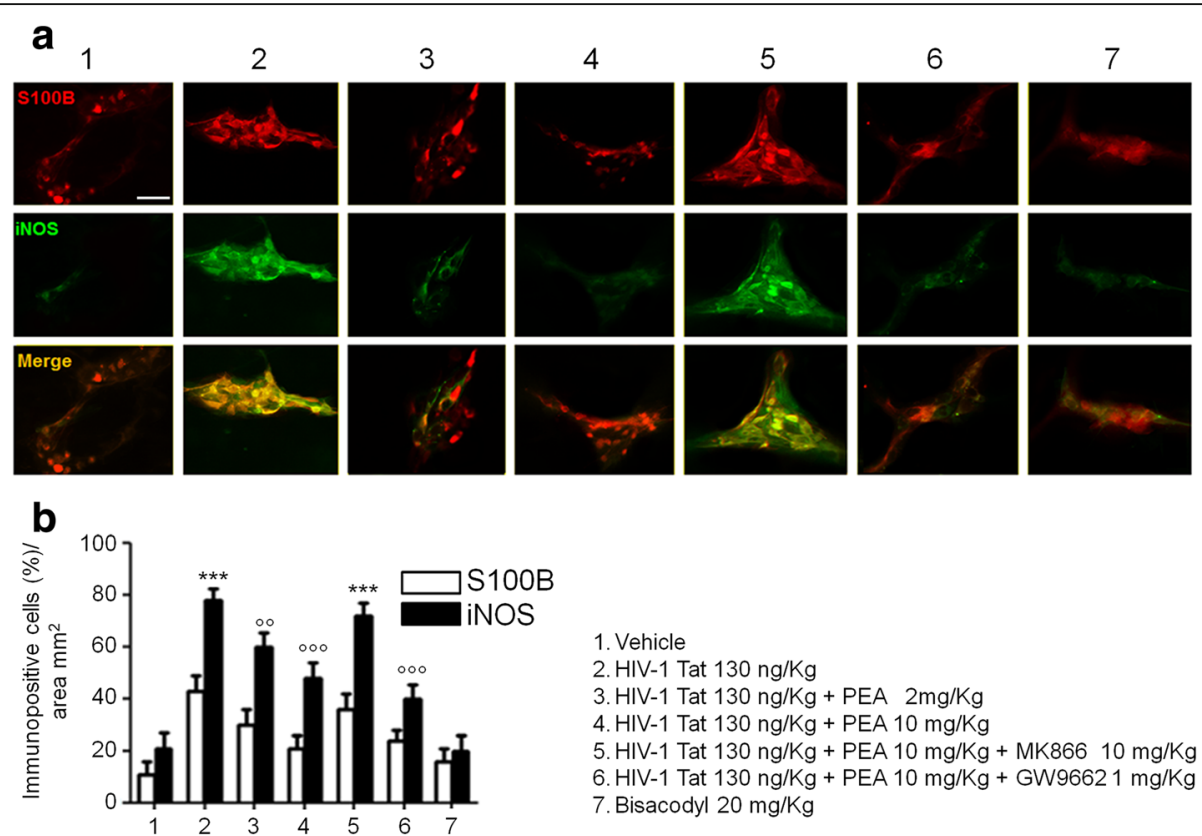

1. Vehicle
2. HIV-1 Tat $130 \mathrm{ng} / \mathrm{Kg}$
3. HIV-1 Tat $130 \mathrm{ng} / \mathrm{Kg}+$ PEA $2 \mathrm{mg} / \mathrm{Kg}$
4. HIV-1 Tat $130 \mathrm{ng} / \mathrm{Kg}+$ PEA $10 \mathrm{mg} / \mathrm{Kg}$
5. HIV-1 Tat $130 \mathrm{ng} / \mathrm{Kg}+$ PEA $10 \mathrm{mg} / \mathrm{Kg}+\mathrm{MK} 86610 \mathrm{mg} / \mathrm{Kg}$
6. HIV-1 Tat $130 \mathrm{ng} / \mathrm{Kg}+$ PEA $10 \mathrm{mg} / \mathrm{Kg}+\mathrm{GW} 96621 \mathrm{mg} / \mathrm{Kg}$
7. Bisacodyl $20 \mathrm{mg} / \mathrm{Kg}$

Fig. 4 PEA reduces S100B/iNOS expression in submucosal plexi of HIV-1 Tat-treated rat. Immunofluorescence analysis showing that HIV-1 Tat (130 ng/Kg) induced a marked increase of S100B and iNOS expression in submucosal plexi that was reduced by PEA through a PPARa-dependent mechanism. Bisacodyl (20 mg/kg) administration failed to significantly affect S100B/iNOS co-expression. a The panel shows S100B (red) and iNOS (green) immunoreactivity and $\mathbf{b}$ their respective quantification (open and filled bars indicate S100B and iNOS expression, respectively). The results are expressed as mean \pm SEM of $n=5$ experiments. ${ }^{* *} p<0.001$ vs. vehicle group; ${ }^{\circ 0} p<0.01$ and ${ }^{000} p<0.001$ vs. HIV-1 Tat group. Scale bar $=20 \mu \mathrm{m}$

toxin and that their modulation is able to reduce HIV-1 Tat diarrheagenic effects by inhibiting the overexpression of S100B and iNOS and of the TLR4/NF-kappaB axis, respectively.

Furthermore, supporting the role of EGCs and their activation in HIV-1 Tat diarrhea, we also observed that when the secretory diarrhea was induced by a nonimmunological stimulus (i.e., bisacodyl), no significant changes in glial network and markers were noticed.

Furthermore, we evaluated whether PEA was able to decrease glial activation and to improve the diarrhea, respectively. PEA has been recently showed to improve colonic inflammation through EGCs/TLR4-dependent PPAR $\alpha$ activation [25]. Here, we demonstrated that PEA administration significantly and dose-dependently counteracted all diarrheal hallmarks in rodents, as shown by the decrease of stool frequency and weight, and by the rescue of water losses in colonic lumen.

According to our previous reports in human and animal models of intestinal inflammation, we also showed that the anti-diarrheal effect of PEA was selectively mediated by PPAR $\alpha$ receptor activation $[25,32]$. In fact, PPAR $\alpha$, but not PPAR $\gamma$ antagonist, significantly inhibited PEA effects, with this being further confirmed in a transgenic PPAR $\alpha$ knockout model. Though HIV-1 Tat administration in these mice-induced secretory diarrhea, the treatment with PEA, even at high doses, did not evoke any anti-diarrheal effect, likely because the site of action was not expressed.
However, it has to be noted that PPAR $\alpha$ receptor sites are expressed by EGCs, as well as by enteric neurons, supporting that the anti-diarrheagenic effects of PEA are more likely to be the result of its synergistic effects on both neurons and glial cells.

According to our previous results, obtained in an experimental model of colitis [25], PEA was able to dampen EGC activation and the consequent overexpression of S100B and iNOS protein in the submucosal plexus isolated from colon. Moreover, we found that PEA, through the selective PPAR $\alpha$ involvement, blocked the TLR4/NF-kappaB activation in the submucosal plexus of rats with HIV-1 Tat-induced diarrhea. These effects caused a decreased activation of EGCs with the reduction of S100B, GFAP, iNOS, and NO expression in the cell milieu. In PPAR ${ }^{-1-}$ mice, PEA failed to prevent HIV-1 Tat-induced EGC activation, further confirming that these effects are mediated by PPAR $\alpha$ receptors.

Since its discovery, PEA has been believed to be an endogenous cannabinoid-like lipid able to suppress inflammatory responses in vitro [33]; moreover, PEA has been described to reduce gastrointestinal motility in mice model of colitis [34]. Also, during intestinal inflammation, PEA level increases, most probably as a protective response to mucosal damage [35]. The observation that PEA levels are higher in colonic mucosa of patients with ulcerative colitis and in experimental models of colitis strengths, the hypothesis that this endogenous compound 


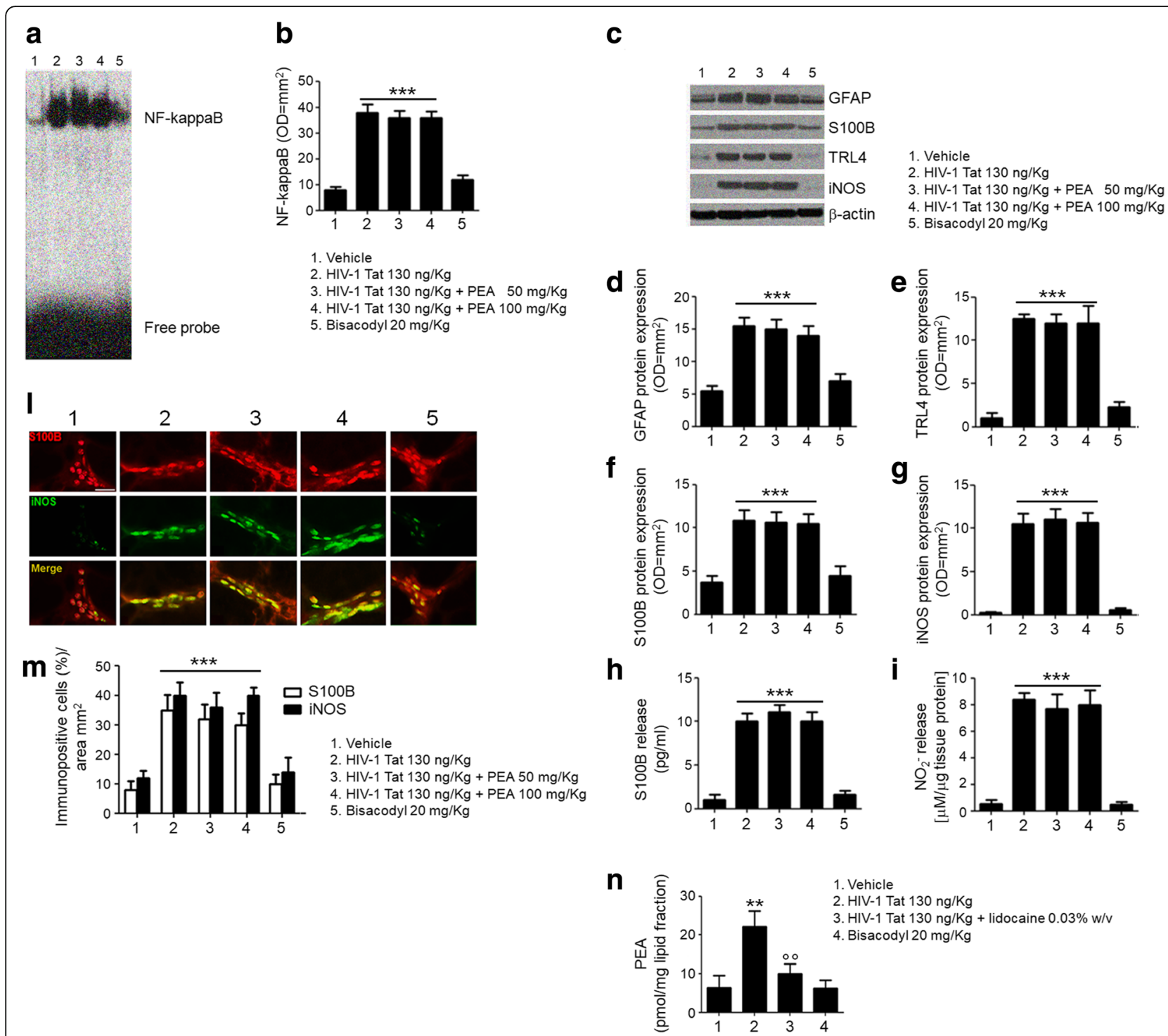

Fig. 5 PEA fails to counteract submucosal plexus-EGC activation induced by HIV-1 Tat in PPARa ${ }^{-1-}$ mice. EMSA analysis showed marked upregulation of NF-kappaB in submucosal plexi at day 7 following HIV-1 Tat administration, compared to vehicle group. PEA was ineffective to reduce NF-kappaB activation in PPARa ${ }^{-1-}$ mice, while bisacodyl $(20 \mathrm{mg} / \mathrm{kg})$ did not induce NF-kappaB upregulation. a Representative NF-kappaB activation complex bands and $\mathbf{b}$ their densitometric quantification (OD = optical density in $\mathrm{mm}^{2}$ ). HIV-1 Tat significantly increased GFAP, S100B, TLR4, and iNOS protein expression and $\mathrm{S1O0B}$ and $\mathrm{NO}_{2}^{-}$levels in submucosal plexi isolated from PPARa ${ }^{-1-}$ mice in the same experimental conditions. Again, HIV-1 Tat-mediated effects were unaffected by PEA, while bisacodyl did not induce any change in the above investigated parameters. $\mathbf{c}$ Representative immunoreactive bands of analyzed proteins, $\mathbf{d}-\mathbf{g}$ their respective densitometric quantification (normalized against the expression of the housekeeping protein $\beta$-actin; $\mathrm{OD}=$ optical densitometry in $\mathrm{mm}^{2}$ ) and $\mathbf{h}, \mathbf{i} \mathrm{S100B}$ and $\mathrm{NO}_{2}^{-}$levels. Immunofluorescence analysis showed an increased S100B/iNOS co-expression in submucosal plexus-EGCS at day 7 after HIV-1 Tat administration, vs. vehicle group. As expected, PEA was unable to counteract HIV-1 Tat activity and bisacodyl did not induce any S100B/iNOS upregulation. I S100B (red) and iNOS (green) immunoreactivity with $\mathbf{m}$ the respective quantification of S100B (open bars) and iNOS (filled bars) expression in the submucosal plexi. $\mathbf{n}$ HIV-1 Tat caused a marked increase of endogenous PEA content in submucosal plexi vs. vehicle group. Lidocaine inhibited HIV-1 Tat activity, whereas bisacodyl did not produce any alteration in endogenous PEA production. The results are expressed as mean \pm SEM of $n=5$ experiments. ${ }^{* *} p<0.01$ and ${ }^{* * *} p<0.001$ vs. vehicle group; ${ }^{\circ 0} p<0.01$ and ${ }^{000} p<0.001$ vs. HIV-1 Tat group. Scale bar $=100 \mu \mathrm{m}$

may act as "on-demand modulator" of inflammatory processes in the gut [36]. Very interestingly, we found that in our models of HIV-1 Tat-induced diarrhea, the levels of endogenous PEA are significantly increased, while in the non-immunologic model of diarrhea obtained via bisacodyl administration, they remained unaltered. The fact that the endogenous level of PEA was significantly reduced by lidocaine indicates that, at least in our experimental 
conditions, PEA acts specifically by following the immune stimulus (HIV-1 Tat protein), behaving like a regulative ALIAmide and such action is intimately modulated by the ENS, and at least partly mediated by EGC activity.

\section{Conclusions}

Although we cannot definitely rule out the role of enteric neuron dysfunction in mediating these effects, our results indicate that EGCs play a role in HIV-1 Tat-induced diarrhea and highlight the importance of these cells in regulating immune/inflammatory response featuring intestinal disturbance occurring in AIDS infection. We also demonstrated that PEA, by targeting HIV-1 Tat-induced neuroinflammatory responses, significantly modulates the diarrhea and that this occurs through the selective PPAR $\alpha$ involvement. If confirmed by clinical trials in humans, our findings suggest that PEA, given its low cost and toxicological profile [37], might be regarded as promising tool that may integrate the current therapeutic approaches for treating a high-morbidity condition like diarrhea in HIVinfected patients.

\section{Additional files}

Additional file 1: PEA failed to inhibit bisacodyl-induced diarrhea in rats. Bisacodyl $(20 \mathrm{mg} / \mathrm{kg})$ caused a significant increase of (a) daily defecation frequency, (b) average daily number of wet spots, and (c) fluid accumulation within 7 days from diarrhea induction, vs. vehicle group. PEA (2-10$50 \mathrm{mg} / \mathrm{kg}$ ) resulted ineffective to exert any anti-diarrheal activity. The results are expressed as mean \pm SEM of $n=5$ experiments. ${ }^{* *} p<0.001$ vs. vehicle group. (TIFF $1347 \mathrm{~kb}$ )

Additional file 2: PEA failed to improve diarrhea course in $\mathrm{PPARa}^{-/}$ mice. HIV-1 Tat (130 ng/Kg)-induced diarrhea in PPARa ${ }^{-/-}$mice, increasing (a) daily defecation frequency, (b) average daily number of wet spots, and (c) fluid accumulation within 7 days from diarrhea induction, vs. vehicle group. PEA (50-100 mg/kg) did not show any significant effect even at highest doses. The results are expressed as mean \pm SEM of $n=5$ experiments. ${ }^{* * *} p<0.001$ vs. vehicle group. (TIFF $580 \mathrm{~kb}$ )

\section{Abbreviations \\ AIDS: Acquired immunodeficiency syndrome; ALIAmides: Autacoid local inflammation antagonism amides; ANOVA: Analysis of variance; EDTA: Ethylenediaminetetraacetic acid; EGCs: Enteric glial cells; ELISA: Enzyme-linked immunosorbent assay; EMSA: Electrophoretic mobility shift assay; ENS: Enteric nervous system; GDNF: Glial cell-derived neutrophic factor; GFAP: Glial fibrillary acidic protein; GSNO: S- nitrosoglutathione; HIV-1: Human immunodeficiency virus-type 1; iNOS: Inducible nitric oxide synthase; LC-MS/MS: Liquid chromatography coupled to tandem mass spectrometry; $\mathrm{NO}$ : Nitric oxide; $\mathrm{NO}_{2}^{-}$: Nitrite; PEA: Palmitoylethanolamide; PPARs: Peroxisome proliferator-activated re- ceptors; PPARa: Peroxisome proliferator-activated receptor $a_{\text {; }}$ PPARa $^{-\prime}$ -: Peroxisome proliferator-activated receptor a knockout; PPARY: Peroxisome proliferator-activated receptor $\gamma_{\text {; }}$ Tat: Trans-activating factor protein; TLR4: Toll-like receptor 4; TLRs: Toll-like receptors}

\section{Acknowledgements}

Not applicable.

\section{Funding}

G.E. and G.S. were partially funded, respectively, by MIUR (PRIN 2009HLNNRL) and La Sapienza University (C26N15YY9F_ 2015).

\section{Availability of data and materials}

Please contact the authors for data requests.

\section{Authors' contributions}

GS and GE conceived and supervised the project. LS, SG, and AD performed most of the experiments and analyses. MP, RL, and JL helped with the analysis and contributed to the writing and revision of the manuscript. LS performed and contributed to immunofluorescence studies. EB and LSt contributed to the critical revision of the manuscript. All the authors discussed the results and commented on the manuscript. All authors read and approved the final version of the manuscript.

\section{Ethics approval}

All procedures were approved by the local University's Ethics Committee. Animal care was in compliance with the IASP and European Community (EC L358/1 18/12/86) guidelines on the use and protection of animals in experimental research and met stipulations of the guide for the care and use of laboratory animals, as well as recommendations of reduction, refinement, and replacement (known as the 3 Rs).

\section{Consent for publication}

Not applicable

\section{Competing interests}

The authors declare that they have no competing interests.

\section{Publisher's Note}

Springer Nature remains neutral with regard to jurisdictional claims in published maps and institutional affiliations.

\section{Author details}

'Department of Clinical Medicine and Surgery, "Federico II" University of Naples, 80131 Naples, Italy. ${ }^{2}$ Department of Physiology and Pharmacology, "Vittorio Erspamer", La Sapienza University of Rome, 00185 Rome, Italy. ${ }^{3}$ Department of Anatomy, China Medical University, Shenyang, Liaoning Province 110122, People's Republic of China. ${ }^{4}$ Department of Translational Medical Science, Section of Pediatrics, University Federico II, 80131 Naples, Italy.

Received: 27 October 2017 Accepted: 9 March 2018

Published online: 24 March 2018

\section{References}

1. Poorolajal J, Hooshmand E, Mahjub H, Esmailnasab N, Jenabi E. Survival rate of AIDS disease and mortality in HIV-infected patients: a meta-analysis. Public Health. 2016;139:3-12.

2. Zacharof A. AIDS-related diarrhea-pathogenesis, evaluation and treatment. Ann Gastroenterol. 2001;14:22-6.

3. Siddiqui U, Bini EJ, Chandarana K, Leong J, Ramsetty S, Schiliro D, et al. Prevalence and impact of diarrhea on health-related quality of life in HIVinfected patients in the era of highly active antiretroviral therapy. J Clin Gastroenterol. 2007:41:484-90.

4. Goldstein G. HIV-1 Tat protein as a potential AIDS vaccine. Nat Med. 1996;1: 960-4.

5. Buccigrossi V, Laudiero G, Nicastro E, Miele E, Esposito F, Guarino A. The HIV-1 transactivator factor (Tat) induces enterocyte apoptosis through a redox-mediated mechanism. PLoS One. 2011;6:e29436.

6. Berni Canani R, Cirillo P, Mallardo G, Buccigrossi V, Secondo A, Annunziato L, et al. Effects of HIV-1 Tat protein on ion secretion and on cell proliferation in human intestinal epithelial cells. Gastroenterology. 2003;124:368-76.

7. Ngwainmbi J, De DD, Smith TH, El-Hage N, Fitting S, Kang M, et al. Effects of HIV-1 Tat on enteric neuropathogenesis. J Neurosci. 2014;34:14243-51.

8. Galligan JJ. HIV, opiates, and enteric neuron dysfunction. Neurogastroenterol Motil. 2015;27:449-54.

9. Aubé A, Cabarrocas J, Bauer J, Philippe D, Aubert P, Doulay F, et al. Changes in enteric neurone phenotype and intestinal functions in a transgenic mouse model of enteric glia disruption. Gut. 2006;55:630-8.

10. Nasser Y, Fernandez E, Keenan CM, Ho W, Oland LD, Tibbles LA, et al. Role of enteric glia in intestinal physiology: effects of the gliotoxin fluorocitrate on motor and secretory function. Am J Physiol Gastrointest Liver Physiol. 2006;291:G912-27. 
11. Capoccia E, Cirillo C, Gigli S, Pesce M, D’Alessandro A, Cuomo R, et al. Enteric glia: a new player in inflammatory bowel diseases. Int J Immunopathol Pharmacol. 2015;28:443-51.

12. Barajon I, Serrao G, Arnaboldi F, Opizzi E, Ripamonti G, Balsari A, et al. Tolllike receptors 3, 4, and 7 are expressed in the enteric nervous system and dorsal root ganglia. J Histochem Cytochem. 2009;57:1013-23.

13. Turco F, Sarnelli G, Cirillo C, Palumbo I, De Giorgi F, D'Alessandro A, et al. Enteroglial-derived S100B protein integrates bacteria-induced Toll-like receptor signalling in human enteric glial cells. Gut. 2014;63:105-15.

14. Cirillo C, Sarnelli G, Turco F, Mango A, Grosso M, Aprea G, et al. Proinflammatory stimuli activates human-derived enteroglial cells and induces autocrine nitric oxide production. Neurogastroenterol Motil. 2011;23:e372-82.

15. Ruhl A, Franzke S, Collins SM, Stremmel W. Interleukin-6 expression and regulation in rat enteric glial cells. Am J Physiol Gastrointest Liver Physiol. 2001;280:G1163-71.

16. Savidge TC, Newman P, Pothoulakis C, Ruhl A, Neunlist M, Bourreille A, et al. Enteric glia regulate intestinal barrier function and inflammation via release of S-nitrosoglutathione. Gastroenterology. 2007;132:1344-58.

17. Zhang DK, He FQ, Li TK, Pang XH, Cui DJ, Xie Q, et al. Glial-derived neurotrophic factor regulates intestinal epithelial barrier function and inflammation and is therapeutic for murine. J Pathol. 2010;222:213-22.

18. Van Landeghem L, Mahé MM, Teusan R, Léger J, Guisle I, Houlgatte R, et al. Regulation of intestinal epithelial cells transcriptome by enteric glial cells: impact on intestinal epithelial barrier functions. BMC Genomics. 2009;10:507.

19. Cirillo C, Sarnelli G, Esposito G, Grosso M, Petruzzelli R, lzzo P, et al. Increased mucosal nitric oxide production in ulcerative colitis is mediated in part by the enteroglial-derived S100B protein. Neurogastroenterol Motil. 2009;21:1209-e112.

20. Esposito G, Cirillo C, Sarnelli G, De Filippis D, D'Armiento FP, Rocco A, et al. Enteric glial-derived S100B protein stimulates nitric oxide production in celiac disease. Gastroenterology. 2007;133:918-25.

21. Esposito G, Capoccia E, Gigli S, Pesce M, Bruzzese E, Alessandro AD, et al. HIV-1 Tat-induced diarrhea evokes an enteric glia-dependent neuroinflammatory response in the central nervous system. Sci Rep. 2017;7:7735.

22. Calignano A, La Rana G, Piomelli D. Antinociceptive activity of the endogenous fatty acid amide, palmitylethanolamide. Eur J Pharmacol. 2001; 419:191-8.

23. Lambert D, Vandervoorte S, Diependaele G, Govaerts S, Robert A. Anticonvulsant activity of $\mathrm{N}$-palmitoylethanolamide, a putative endocannabinoid, in mice. Epilepsia. 2001;42:321-7.

24. Lo Verme J, Fu J, Astarita G, La Rana G, Russo R, Calignano A, et al. The nuclear receptor peroxisome proliferator-activated receptor-alpha mediates the anti-inflammatory actions of palmitoylethanolamide. Mol Pharmacol. 2005;67:15-9.

25. Esposito G, Capoccia E, Turco F, Palumbo I, Lu J, Steardo A, et al. Palmitoylethanolamide improves colon inflammation through an enteric glia/toll like receptor 4-dependent PPAR-a activation. Gut. 2014;63:1300-12.

26. Ateufack G, Nana Yousseu W, Dongmo Feudjio B, Fonkeng Sama L, Kuiate J, Kamanyi A. Antidiarrheal and in vitro antibacterial activities of leaves extracts of hibiscus asper. Hook. f. (malvaceae). Asian J Pharm Clin Res. 2014;7:130-6.

27. Ghafouri N, Larsson B, Turkina MV, Karlsson L, Fowler CJ, Gerdle BGB. High levels of N-palmitoylethanolamide and N-stearoylethanolamide in microdialysate samples from myalgic trapezius muscle in women. PLoS One. 2011;6:e27257.

28. Weber R. Enteric infections and diarrhea in human immunodeficiency virusinfected persons. Archives Inter Med. 1999;159:1473.

29. Nazli A, Chan O, Dobson-belaire WN, Ouellet M, Tremblay MJ, Scott D, et al, Exposure to HIV-1 directly impairs mucosal epithelial barrier integrity allowing microbial translocation. PLoS Pathog. 2010;6:e1000852.

30. Fitting S, Ngwainmbi J, Kang M, Khan F a, Stevens DL, Dewey WL, et al. Sensitization of enteric neurons to morphine by HIV-1 Tat protein. Neurogastroenterol Motil. 2015;27:468-80.

31. Lundgren O, Peregrin AT, Persson K, Kordasti S, Uhnoo I, Svensson L. Role of the enteric nervous system in the fluid and electrolyte secretion of rotavirus diarrhea. Science. 2000;287:491-6.

32. Sarnelli G, Alessandro AD, luvone T, Capoccia E, Gigli S, Pesce M, et al. Palmitoylethanolamide modulates inflammation-associated vascular endothelial growth factor (VEGF) signaling via the Akt/mTOR pathway in a selective peroxisome proliferator-activated receptor alpha (PPAR-a)dependent manner. PLoS One. 2016;11:e0156198.

33. De Filippis D, Negro L, Vaia M, Cinelli MP, luvone T. New insights in mast cell modulation by palmitoylethanolamide. CNS Neurol Disord Drug Targets. 2013;12:78-83
34. Capasso R, Izzo AA, Fezza F, Pinto A, Capasso F, Mascolo N, et al. Inhibitory effect of palmitoylethanolamide on gastrointestinal motility in mice. $\mathrm{Br} \mathrm{J}$ Pharmacol. 2001;134:945-50

35. De Filippis D, D'Amico A, Cipriano M, Petrosino S, Orlando P, Di Marzo V, et al. Levels of endocannabinoids and palmitoylethanolamide and their pharmacological manipulation in chronic granulomatous inflammation in rats. Pharmacol Res. 2010;61:321-8.

36. Costa B, Comelli F, Bettoni I, Colleoni M, Giagnoni G. The endogenous fatty acid amide, palmitoylethanolamide, has anti-allodynic and anti-hyperalgesic effects in a murine model of neuropathic pain: involvement of CB(1), TRPV1 and PPARgamma receptors and neurotrophic factors. Pain. 2008;139:541-50.

37. Petrosino S, Cristino L, Karsak M, Gaffal E, Ueda N, Tüting T, et al. Protective role of palmitoylethanolamide in contact allergic dermatitis. Allergy. 2010;65:698-711.

\section{Submit your next manuscript to BioMed Central and we will help you at every step:}

- We accept pre-submission inquiries

- Our selector tool helps you to find the most relevant journal

- We provide round the clock customer support

- Convenient online submission

- Thorough peer review

- Inclusion in PubMed and all major indexing services

- Maximum visibility for your research

Submit your manuscript at www.biomedcentral.com/submit
) Biomed Central 